\title{
Guest editorial: Special issue on optimisation in multi-agent systems
}

\author{
Sarvapali D. Ramchurn • Alessandro Farinelli • \\ Juan A. Rodríguez Aguilar
}

Published online: 27 June 2010

(C) The Author(s) 2010

Multi-agent systems applications in a number of areas such as e-commerce, disaster management, and information acquisition through embedded devices (e.g. wireless sensor networks) have generated a number of new challenges for algorithm designers. These challenges mainly take the form of very hard optimisation problems that are substantially different from problems traditionally dealt with in other areas (e.g. industrial processes or scheduling applications). More specifically, novel challenges come from the distributed nature of multiagent systems where the actors reside on different computational units and can communicate only a limited amount of information with their neighbours. Moreover, the agents may be acting on behalf of different stakeholders each with its own aims and objectives, have different computation/communication capabilities, and be tied to physical devices prone to failures. Moreover, given the dynamic nature of the application scenarios, effective algorithms have to provide anytime solutions and approximate techniques are often required/desirable.

This special issue collects a selection of contributions that focus on optimisation techniques for multi-agent systems. Preliminary version of these contributions were presented at the OptMAS 2009 and 2008 workshops, which were held in conjunction with the International Conference on Autonomous Agents and Multi-agent Systems. The workshops provided a multi-disciplinary forum for multi-agent algorithm designers, complementing the wide spectrum of knowledge required to successfully address the challenges outlined above.

The contributions published in this special issue focus on several key aspects of these challenges and provide a range of perspectives on the common problem of providing effective and efficient techniques to solve hard optimisation problems in a multi-agent context. Specifically, this issue includes the following contributions:

- Benchmarking Hybrid Algorithms for Distributed Constraint Optimisation Games by A. C. Chapman, A. Rogers, and Nicholas R. Jennings-this contribution relates solution algorithms for Distributed Constraint Optimisation Problems using game theory. Specifically, DCOP techniques are analysed using a potential game characterisation and novel hybrid algorithms are proposed and empirically analysed.

S. D. Ramchurn (凶) · A. Farinelli · J. A. R. Aguilar

University of Southampton, Southampton, UK

e-mail: sdr@ecs.soton.ac.uk 
- Optimising coalition formation for tasks with dynamically evolving rewards and nondeterministic action effects by M. A. Khan, D. Turgut, and L. Boloni-this article focusses on coalition formation in dynamic uncertain environments. Specifically, this contribution proposes a formulation of the coalition formation problem based on Markov Decision Processes, and applies this approach to the problem of coordinating rescue operations in emergency scenarios.

- Constructing a unifying theory of dynamic programming DCOP algorithms via the Generalized Distributive Law by M. Vinyals, J. A. Rodriguez-Aguilar, and J. Cerquides-this contribution proposes a novel message passing algorithm, Action-GDL, to efficiently solve Distributed Constraint Optimisation Problems. Specifically, the authors show how Action-GDL provides a unifying framework for many well-known DCOP solution techniques (such as DPOP and DCPOP) and how, using particular heuristics, Action-GDL can outperform those approaches.

- Towards efficient multiagent task allocation in the RoboCup Rescue: a biologically inspired approach by F. dos Santos and A. Bazzan-this study focusses on team formation for coordinating rescue operations. The authors propose a biologically inspired approximate algorithm that is able to efficiently solve the task assignment problem faced by teams of emergency responders and evaluate their approach in the RoboCup Rescue Simulation environment.

The breadth and depth of the above approaches is witness to the fact that solving multi-agent optimisation problems cannot be tackled by one single technique or framework. Hence, it is hoped that this collection of articles will build the initial steps towards a more concerted effort from experts in different research areas (e.g. operations research, signal processing, machine learning, and game theory). 